\title{
Comparing the Efficacy of Urinary hCG vs Recombinant hCG for Final Maturation of Oocyte in GnRH Antagonist IVF/ICSI Cycle
}

\author{
G Ashwini Sidhmalswamy, MS Srinivas, K Dipika, K Anu, Kamini A Rao, D Mekhala
}

\begin{abstract}
Aims and objectives: To compare the efficacy of urinary human chorionic gonadotropin (uhCG) vs recombinant human chorionic gonadotropin (rhCG) for triggering ovulation in gonadotropinreleasing hormone $(\mathrm{GnRH})$ antagonist in vitro fertilization (IVF) cycle.
\end{abstract}

Materials and methods: Two hundred patients who underwent GnRH antagonist IVF/ICSI cycles were analyzed, 100 of them received recombinant hCG $250 \mu \mathrm{g}$ (injection overtrelle) and another group of 100 received urinary hCG (injection ovutrig) either $5000 \mathrm{IU}$ or $10,000 \mathrm{IU}$ for final oocyte maturation and ovulation trigger. The primary outcome measured was total oocytes retrieved and the secondary outcome measured 09 was the maturity rate fertilization rate, cleavage rate and clinical pregnancy rates were also compared.

Results: Age, day 2 follicle stimulating hormone (FSH) and total dose of rFSH were comparable between the groups. There were no significant differences between the groups in terms of the mean number of oocytes retrieved per follicle (uhCG; 12.5, rhCG; 12.09 , p-value-0.6698). Similarly, there was statistically no difference in any of the other parameters studied between the two groups. That is number of mature oocytes, number of fertilized oocytes and number of cleaved oocytes, as the $p$-value was not significant. The clinical pregnancy rate was somewhat higher in the uhCG group but was not statistically significant.Both treatments were well tolerated and there was no significant side effects for either drug.

Conclusion: There is no difference in clinical outcomes between urinary and recombinant hCG for induction of final oocyte maturation. Additional factors, including the cost, drug availability and ease of administration, should be considered, when choosing gonadotropin type.

Keywords: Urinary hCG, Recombinant hCG, Final oocyte trigger.

How to cite this article: Sidhmalswamy GA, Srinivas MS, Dipika K, Anu K, Rao KA, Mekhala D. Comparing the Efficacy of Urinary hCG vs Recombinant hCG for Final Maturation of Oocyte in GnRH Antagonist IVF/ICSI Cycle. Int J Infertility Fetal Med 2012;3(3):92-96.

Source of support: Nil

Conflict of interest: None declared

\section{INTRODUCTION}

The human chorionic gonadotropin (hCG) is a member of the glycoprotein hormone family. This hormone consists

Date of Received: 24-05-12

Date of Acceptance: 09-08-12

Date of Publication: September 2012 of a noncovalently bound $\alpha$ and $\alpha$ subunits. The $\alpha$ subunit is identical within a species and $\beta$ subunit is unique and hormone specific. The $\beta$ subunit of hCG is encoded by multiple genes on chromosome 19 adjacent to the structurally related luteinizing hormone (LH) $\beta$ subunit gene.

The hCG has been used to mimic the endogenous LH surge as there are considerable structural similarities between hCG and human (h) LH, and hence both hormones stimulate the same receptor. ${ }^{1}$ Physiologically, the preovulatory surge LH causes the final maturation of oocytes and induces ovulation. The hCG is readily available in the urine of pregnant women, whereas only low concentrations of LH are found in the urine of postmenopausal women. Hence, urinary-derived hCG (uhCG) has been used as an alternative to LH to induce final oocyte maturation in women undergoing ovarian stimulation for in vitro fertilization (IVF) or intracytoplasmic sperm injection (ICSI). ${ }^{2}$

The uhCG has certain limitations, such as batch to batch inconsistency, urinary protein contamination, and scarcity of the available biosource. In contrast, recombinant hCG (rhCG) is manufactured with a high degree of purity and high specificity, and it is practically free from fetal bovine serum proteins, nucleic acids or other contaminants. ${ }^{3,4}$ The rhCG is derived from genetically engineered Chinese hamster ovary cells through recombinant DNA technology. This product has a high purity that facilitates characterization and quantitation by physicochemical means, reducing the need for animal bioassays, rhCG has been shown to stimulate luteal function in primates ${ }^{5}$ and to stimulate final follicular maturation, luteinization and ovulation in women undergoing assisted reproduction technology ${ }^{6}$ or controlled ovarian stimulation for ovulation induction. ${ }^{7}$

Several randomized trials have tested the efficacy of uhCG vs rhCG for final maturation of oocyte in GnRH agonist downregulated IVF cycles, finding equivalence between them. We wanted to study the same outcome in GnRH antagonist IVF/ICSI cycle as there are not many studies using this protocol.

So, the objective of present study was to compare the effect of uhCG vs rhCG for triggering ovulation in GnRH antagonist IVF/ICSI cycle. 


\section{MATERIALS AND METHODS}

This prospective study was conducted between January 2011 to July 2011 at Bangalore Assisted Conception Centre, Bengaluru, India.

\section{Patient Selection}

All infertile women aged between 20 and 37 years with indication for IVF/ICSI and undergoing antagonist cycle protocol were included in the study. Inclusion criteria were: Women with (1) regular menstrual cycles of 25 to 35 days, (2) at least a 2-year history of infertility attributing to either tubal factor, AFS grades I and II endometriosis, severe male factor or unexplained factor, (3) FSH on day 2 of the cycle $<12 \mathrm{IU} / \mathrm{ml}$, (4) presence of both the ovaries with normal uterine cavity. Exclusion criteria: (1) Previous history of OHSS, (2) previous history of poor response, (3) BMI >30 $\mathrm{kg} / \mathrm{m}^{2}$, (4) $>3$ previous IVF attempts. Informed consent of the couple was taken. Medical and gynecological history taken. Physical examination with hematology and endocrine screening was done. Hormonal analysis was done by electrochemiluminescent immunoassay. The study protocol was approved by the Institutional Review Board or Ethics Committee.

\section{Treatment}

Standard assisted antagonist protocol was used for ovarian stimulation.

The ovarian stimulation was performed using a recombinant FSH/GnRH-antagonist protocol. ${ }^{8}$ The stimulation was started on the day 2 or 3 of the cycle. The initial gonadotropin dose was predefined at 150 IU (GonalF; Merck Serono, Geneva, Switzerland) for all patients and remained fixed for 4 days; from next day onward, a GnRH antagonist was coadministered (Cetrotide; Merck Serono). Ultrasound and, if required, serum E2 measurements were used to monitor the follicle growth. Stimulation was continued until the criteria of induction of ovulation was met, i.e. atleast three follicles of $\geq 18 \mathrm{~mm}$ size follicle on ultrasonography was present, at this point, final oocyte maturation was induced with either 5000 IU or 10,000 IU uhCG (Pregnyl; NV Organon, Oss, The Netherlands), or $250 \mathrm{mg}$ recombinant hCG (Ovitrelle; Merck Serono). Progesterone in oil, $50 \mathrm{mg}$ IM daily, was used to provide luteal support in all patients.

Oocytes were retrieved 34 to 35 hours after hCG administration, according to the usual procedures. Oocytes after retrival were assessed and inseminated/injected in vitro and upto 2 to 3 embryos were replased 2 to 3 days later. Luteal support was administered in the form of $800 \mathrm{mg} /$ day micronized progesterone vaginally till 2 weeks. Blood sampling for $\beta$-hCG was performed after 2 weeks of embryo transfer followed by an ultrasound scan around day 42 . A clinical pregnancy was defined as the presence of a heart beat at 7 weeks' gestation. Sperm preparation, IVF, intracytoplasmic sperm injection procedures and embryo culture were performed as described elsewhere. ${ }^{9}$

\section{Study End Points Measured}

Primary outcome was comparison of total oocytes retrived per patient between the groups. Secondary outcome was comparison of total number of mature oocytes, number of fertilized oocytes, number of cleaved oocytes and the clinical pregnancy rate between the groups.

\section{STATISTICAL ANALYSIS}

Statistical analysis was done by using unpaired t-test on continuous scale between two groups. Chi-square test/ Fischer exact test was used for study parameters on categorical scale. A p $<0.05$ was considered significant. Software used GraphPad InStat version 3.

\section{RESULTS}

A total of 202 patients were enrolled in the study, of these 200 were analyzed other two patient were not included due to inadequate ovarian response. Of these 200 patients, 100 received uhCG and other 100 received rhCG. None of the cycles was canceled during stimulation and all cycles ended in embryo transfer.

There was no difference in the mean age, day 2 FSH and the mean dose of recombinant FSH and HMG used in both the groups. Mean days of stimulation was similar in both the groups (12 days), as shown in Table 1 .

For the number of oocytes retrieved the two-sided 95\% CI $(-2.35$ to 1.15$)$ fell within the limit predefined for the study $(-3,+3)$, thus conferming the equivalence of the two groups, as shown in Table 2.

Similarly, as shown in Table 3, there was statistically no difference in any of the other parameters studied between the two groups that is number of mature oocytes, number of fertilized oocytes and number of cleaved oocytes, as the p-value was not significant.

Clinical pregnancy rate: Forty-five percent (45/100) were pregnant in uhCG and 42\% (42/100) were pregnant in rhCG group. Pregnancy rate was not statistically significant (p-value: 0.7754), as shown in Graph 1.

Table 1: Demographic and stimulation characteristics

\begin{tabular}{lrr} 
Parameters & \multicolumn{1}{c}{ uhCG } & \multicolumn{1}{c}{ rhCG } \\
\hline Age (yrs) & $31 \pm 4$ & $32 \pm 4$ \\
Day 2 FSH (IU/L) & $6.7 \pm 1.6$ & $6.7 \pm 1.8$ \\
rFSH (IU) & $1621.75 \pm 495.3$ & $1514.60 \pm 466.8$ \\
HMG (IU) & $497 \pm 523.7$ & $551 \pm 735.2$ \\
\hline
\end{tabular}




\begin{tabular}{|c|c|c|c|c|c|c|}
\hline \multicolumn{7}{|c|}{ Table 2: Number of oocytes retrieved } \\
\hline Parameter & \multicolumn{2}{|c|}{ uhCG } & \multicolumn{2}{|c|}{$r h C G$} & \multirow[t]{2}{*}{$\mathrm{p}$-value } & \multirow[t]{2}{*}{$95 \% \mathrm{Cl}$} \\
\hline & Mean (SD) & Median (SD) & Mean (SD) & Median (SD) & & \\
\hline Oocytes retrieved & $12.5(6.9)$ & 11.5 & $12.09(6.9)$ & 11 & 0.6698 & $-2.35-1.51$ \\
\hline
\end{tabular}

\begin{tabular}{|c|c|c|c|c|c|}
\hline \multirow[t]{2}{*}{ Parameters } & \multicolumn{2}{|c|}{$u h C G$} & \multicolumn{2}{|c|}{$r h C G$} & \multirow[t]{2}{*}{$p$-value } \\
\hline & Mean (SD) & Median (SD) & Mean (SD) & Median (SD) & \\
\hline No. of mature oocytes & $9.65(18.7)$ & 8 & $9.19(17.9)$ & 8 & 0.5683 \\
\hline No. of fertilized oocytes & $7.91(18.9)$ & 7 & $7.08(22.6)$ & 6 & 0.2457 \\
\hline No. of cleaved oocytes & $7.73(10.4)$ & 7 & $6.82(17.8)$ & 6 & 0.1876 \\
\hline
\end{tabular}

As shown in Graph 2, incidence of OHSS was 24\% (24/100) in uhCG and 30\% (30/100) in rhCG. The incidence of OHSS between the two groups was not statistically significant (p-value: 0.425).

Early onset OHSS in uhCG was 83.33\% (20/24) and, in rhCG, it was $86.66 \%(26 / 30)$. Late onset OHSS in uhCG was $16.66 \%$ (4/24) and, in rhCG, it was $13.33 \%(4 / 30)$, as shown in Graph 3.

Out of 24 cases of OHSS in uhCG group, nine had moderate OHSS and the remaining were mild OHSS cases, there was no severe case of OHSS reported. In rhCG group, out of 30 OHSS cases, one had severe OHSS, 10 had moderate OHSS and remaining had mild OHSS.

Local tolerance to the injections was good, and similar across the two treatment groups. For each local reaction assessed (itching, redness, swelling, bruising or pain), 97\% produced no reaction others produced only a mild reaction.

\section{DISCUSSION}

This study demonstrated clinical and statistical equivalence of recombinant and uhCG for induction of final follicular maturation. The primary end point used in this study, that is number of oocytes retrieved per patient receiving hCG, was one of the number of end points available to assess hCG efficacy.

In IVF cycles, successful oocyte retrieval is the most direct measure of hCG efficacy as it integrates a cascade of intrafollicular events necessary for ovum release from the follicle. Absence of LH/hCG effect is associated with failed oocyte retrieval or empty follicle syndrome. ${ }^{3,4}$ hCG induces a number of critical physiologic changes at the time of ovulation and subsequent fertilization. These can be broadly categorized as induction of follicular rupture, luteinization and resumption of meiosis. ${ }^{10,11}$

All the parameters like number of oocyte retrieved, oocyte matured, oocytes fertilized, oocytes cleaved and clinical pregnancy rates were comparable between the groups. Several randomized trials have tested the efficacy of uhCG vs rhCG in GnRH agonist downregulated IVF cycles, finding equivalence. ${ }^{12-15}$ Our study was done in GnRH antagonist cycle finding equivalence in the outcomes between urinary and recombinant hCG for induction of final oocyte maturation. The safety of rhCG was comparable with that of the urinary product in terms of clinical assessments and the incidence of serious adverse events.

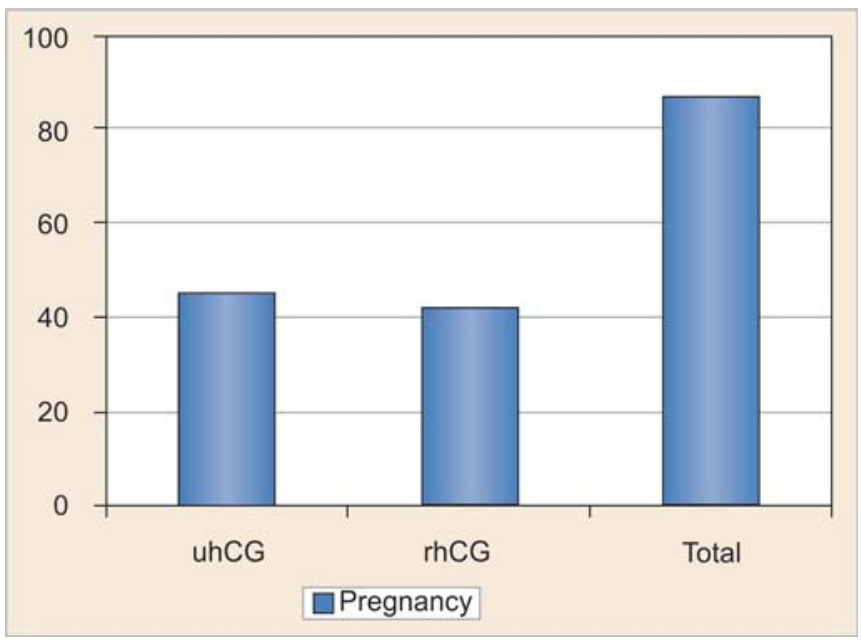

Graph 1: Pregnancy outcome between the groups

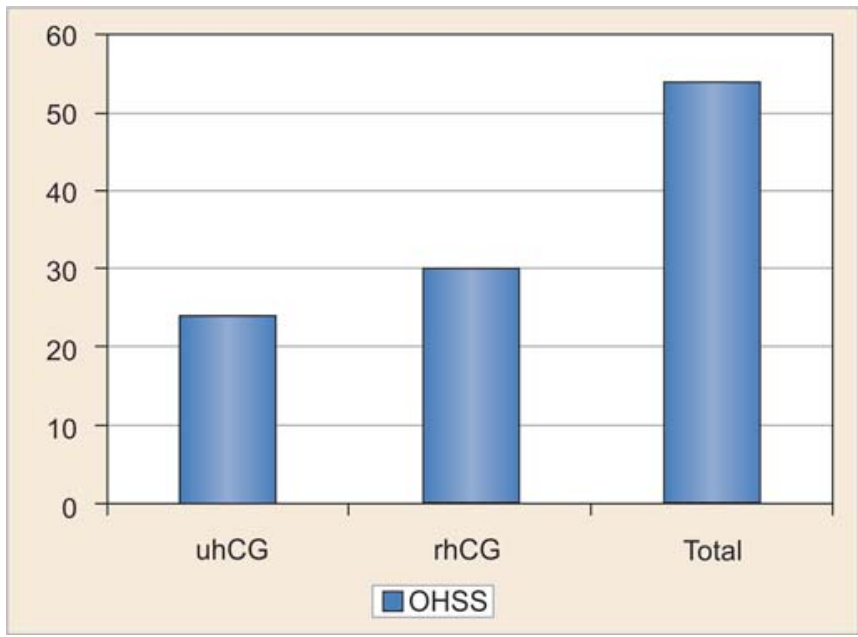

Graph 2: OHSS outcome between the groups 


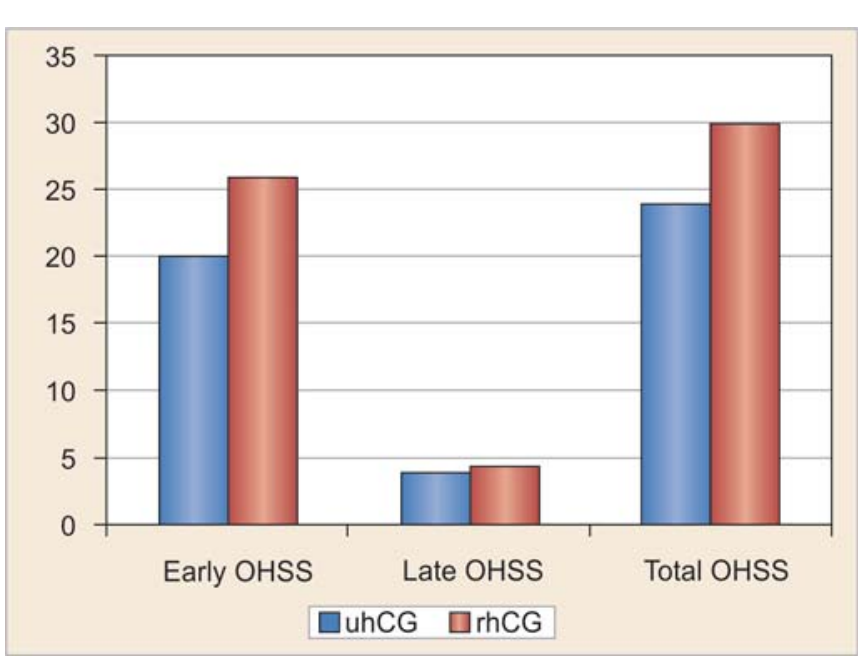

Graph 3: Early and late onset OHSS between the groups

Though pregnancy rates were comparable between treatment groups in our study, in one of the study done by Papanikolaou et al in GnRH antagonist IVF cycles showed higher birth rate after recombinant hCG triggering compared with urinary-derived hCG. ${ }^{16}$ As per the authors, the reason for the difference in the reproductive outcome observed was endometrial advancement of $\geq 3$ days, when treated with the standard 10,000 IU uhCG compared with $250 \mathrm{mg}$ recombinant $\mathrm{hCG},{ }^{17}$ as it is known that when endometrial advancement exceeds 3 days, the probability of pregnancy becomes poor. ${ }^{18}$

This is the first study comparing the efficacy of uhCG and rhCG in GnRH antagonist IVF/ICSI cycle on Indian population. The limitation of our study was it was not a randomized study, though the sample size was good. However, further randomized controlled trials are required in antagonist IVF cycles for firm conclusion.

\section{CONCLUSION}

There is no difference in clinical outcomes between urinary and recombinant hCG for induction of final oocyte maturation. Additional factors including the cost, drug availability and ease of administration should be considered, when choosing gonadotropins.

\section{REFERENCES}

1. Pierce JG, Parsons TF. Glycoprotein hormones (structure and function). Annu Rev Biochem 1981;50:465-95.

2. Gemzell C. Induction of ovulation with human gonadotropins. Recent Prog Horm Res 1965;21:179-204.

3. Zegers-Hochschild F, Fernandez E, Mackenna A, Fabres C, Altieri E, Lopez T. The empty follicle syndrome: A pharmaceutical industry syndrome. Hum Repord 1996;10:2262-65.

4. Penarrubia J, Balasch J, Fabregues F, Crues M, Civico S, Vanrell JS. Recurrent empty follicle syndrome successfully treated with recombinant human chorionic gonadotropin. Hum Reprod 1999;14:1703-06.
5. Duffy DM, Hutchison JS, Stewart DR, Stouffer RL. Stimulatioln of primate luteal function by recombinant human chorionic gonadotropin and modulation of steroid, but not relaxin, production by an inhibitor of 3 beta-hydroxysteroid dehydrogenase during simulated early pregnancy. J Clin Endocrinol Metab 1996;81:2307-13.

6. ERHCG Group: The European Recombinant Human Chorionic Gonadotrophin Study Group. Induction of final follicular maturation and early luteinization in women undergoing ovulation induction for assisted reproduction treatmentrecombinant hCG versus urinary hCG. Human Reprod 2000; 15(7):1446-51.

7. Driscoll GL, Tyler JP, Hangan JT, Fisher PR, Birdsall MA, Knight DC. A prospective, randomized, controlled, double-blind, double-dummy comparison of recombinant and urinary hCG for inducing oocyte maturation and follicular luteinization in ovarian stimulation. Human Reprod 2000;15(6):1305-10.

8. Papanikolaou EG, Bourgain C, Kolibianakis E, Tournaye H, Devroey P. Steroid receptor expression in late follicular phase endometrium in GnRH antagonist IVF cycles is already altered, indicating initiation of early luteal phase transformation in the absence of secretory changes. Hum Reprod 2005;20:1541-47.

9. Papanikolaou EG, CamusM, Kolibianakis EM, VanLanduyt L, Van Steirteghem A, Devroey P. In vitro fertilization with single blastocyst-stage versus single cleavage-stage embryos. N Engl J Med 2006;35:1139-46.

10. Hoff JD, Quigle ME, Yen SSC. Hormonal dynamics at midcycle (a re-evaluation). J Clin Endocrinol Metab 1983;57:792-95.

11. Speroff L, Glass RH, Kase NG. Clinical gynecologic endocrinology and infertility (6th ed). Baltimore: Lippincott Williams and Wilkins 1999:91-97.

12. Chang P, Kenley S, Burns T, Denton G, Currie K, DeVaneG, et al. Recombinant human chorionic gonadotropin (rhCG) in assisted reproductive technology: Results of a clinical trial comparing two doses of rhCG (Ovidrel) to urinary hCG (Profasi) for induction of final follicular maturation in in vitro fertilization embryo transfer. Fertility Sterility 2001;76(1):67-74.

13. ERLH Group: The European Recombinant LH Study Group. Recombinant human luteinizing hormone is as effective as, but safer than urinary human chorionic gonadotropin in inducing final follicular maturation and ovulation in in vitro fertilization procedures: Results of a multicenter double-blind study. J Clin Endocrinol Metabol 2001;86(6):2607-18.

14. Farrag A, Costantini A, Manna C. Recombinant hCG for triggering ovulation increases the rate of mature oocytes in women treated for ICSI. J Assist Reprod Genetics 2008; 25(9-10):461-66.

15. Kovacs P, Kovats T, Bernard A. Comparison of serum and follicular fluid hormone levels with recombinant and urinary human chorionic gonadotropin during in vitro fertilization. Fertility Sterility 2008;90(6):2133-37.

16. Papanikolaou EG, Fatemi H, Camus M, Kyrou D, Polyzos NP Humaidan P, et al. Higher birth rate after recombinant hCG triggering compared with urinary-derived hCG in singleblastocyst IVF antagonist cycles: A randomized controlled trial. Fertility Sterility 2010;94(7):2902-04.

17. Papanikolaou EG, Bourgain C, Fatemi H, Verpoest W, Polyzos N, De Brabanter A, et al. Endometrial advancement after triggering with recombinant or urinary HCG: A randomized controlled pilot study. Reprod Biomed Online 2010;21(1):50-55.

18. Bourgain C, Devroey P. Histologic and functional aspects of the endometrium in the implantatory phase. Gynecol Obstet Invest 2007;64:131-33. 


\section{ABOUT THE AUTHORS}

\section{G Ashwini Sidhmalswamy (Corresponding Author)}

Consultant, Department of Reproductive Medicine, Bangalore Assisted Conception Centre, Bengaluru, Karnataka, India, e-mail: ashni22@yahoo.com

\section{MS Srinivas}

Scientific Director, Department of Clinical Embryology, Bangalore Assisted Conception Centre, Bengaluru, Karnataka, India

\section{K Dipika}

Consultant, Department of Reproductive Medicine, Bangalore Assisted Conception Centre, Bengaluru, Karnataka, India

\section{K Anu}

Deputy Director, Department of Reproductive Medicine Bangalore Assisted Conception Centre, Bengaluru, Karnataka India

\section{Kamini A Rao}

Medical Director, Department of Reproductive Medicine Bangalore Assisted Conception Centre, Bengaluru, Karnataka India

\section{Mekhala}

Consultant, Department of Reproductive Medicine, Bangalore Assisted Conception Centre, Bengaluru, Karnataka, India 